\title{
Overexpression of RPN2 suppresses radiosensitivity of glioma cells by activating STAT3 signal transduction
}

Changyu $\mathrm{Li}^{1+}$, Haonan Ran${ }^{2 \dagger}$, Shaojun Song ${ }^{1}$, Weisong Liu ${ }^{3}$, Wenhui Zou', Bei Jiang ${ }^{4}$, Hongmei Zhao ${ }^{5}$ and Bin Shao ${ }^{6^{*}}$ (D)

\begin{abstract}
Background: Radiation therapy is the primary method of treatment for glioblastoma (GBM). Therefore, the suppression of radioresistance in GBM cells is of enormous significance. Ribophorin II (RPN2), a protein component of an $\mathrm{N}$-oligosaccharyl transferase complex, has been associated with chemotherapy drug resistance in multiple cancers, including GBM. However, it remains unclear whether this also plays a role in radiation therapy resistance in GBM.

Methods: We conducted a bioinformatic analysis of RPN2 expression using the UCSC Cancer Genomics Browser and GEPIA database and performed an immunohistochemical assessment of RPN2 expression in biopsy specimens from 34 GBM patients who had received radiation-based therapy. We also studied the expression and function of RPN2 in radiation-resistant GBM cells.

Results: We found that RPN2 expression was upregulated in GBM tumors and correlated with poor survival. The expression of RPN2 was also higher in GBM patients with tumor recurrence, who were classified to be resistant to radiation therapy. In the radiation-resistant GBM cells, the expression of RPN2 was also higher than in the parental cells. Depletion of RPN2 in resistant cells can sensitize these cells to radiation-induced apoptosis, and overexpression of RPN2 had the reverse effect. Myeloid cell leukemia 1 (MCL1) was found to be the downstream target of RPN2, and contributed to radiation resistance in GBM cells. Furthermore, STAT3 was found to be the regulator of MCL1, which can be activated by RPN2 dysregulation.

Conclusion: Our study has revealed a novel function of RPN2 in radiation-resistant GBM, and has shown that MCL1 depletion or suppression could be a promising method of therapy to overcome the resistance promoted by RPN2 dysregulation.
\end{abstract}

Keywords: GBM, RPN2, STAT3, Signal transduction

\section{Background}

Glioblastomas (GBM) are tumors of the central nervous system and result in $1.2 \sim 1.4 \times 10^{4}$ deaths in the U.S. alone every year (Hess et al., 2004). The existing treatment methods are usually limited to surgical excision,

\footnotetext{
* Correspondence: oqpm0d@163.com

${ }^{+}$Changyu Li and Haonan Ran both authors contributed equally to this work and should be considered as equal first coauthors.

${ }^{6}$ Neurosurgery, The First Affiliated Hospital of Harbin Medical University, No.199 Dazhi Street, Nangang District, Harbin 150001, Heilongjiang, China Full list of author information is available at the end of the article
}

radiation, and temozolomide chemotherapy (Stupp et al., 2005). Despite these aggressive treatments, survival rates after treatment remain low, at an average of around 1215 months (Preusser et al., 2011). It is thought that GBM tumors can obtain chemo and radiation therapy resistance by stimulating DNA repair systems or by initiating changes to the cell cycle and apoptosis (Mirimanoff et al., 2006; Mrugala \& Chamberlain, 2008). Resistance to chemotherapy has been widely investigated and has mostly focused on the expression of MGMT (0-6 methylguanine-DNA Methyltransferase) (Perazzoli et al.,

(c) The Author(s). 2020 Open Access This article is licensed under a Creative Commons Attribution 4.0 International License, which permits use, sharing, adaptation, distribution and reproduction in any medium or format, as long as you give appropriate credit to the original author(s) and the source, provide a link to the Creative Commons licence, and indicate if changes were made. The images or other third party material in this article are included in the article's Creative Commons licence, unless indicated otherwise in a credit line to the material. If material is not included in the article's Creative Commons licence and your intended use is not permitted by statutory regulation or exceeds the permitted use, you will need to obtain permission directly from the copyright holder. To view a copy of this licence, visit http://creativecommons.org/licenses/by/4.0/. 
2015). However, the fundamental mechanisms underlying radiotherapy resistance and its generation are still unclear. Radiation therapy remains a primary method of treatment for GBM (Ghotme et al., 2017), and therefore the reduction of radioresistance in GBM cells and therapeutic targets is of huge significance.

Ribophorin II (RPN2) is a protein component of an Noligosaccharyl transferase complex, the downregulation of which can trigger apoptosis in human breast cancer cells resistant to docetaxel., and its silencing confers sensitivity of the tumor to cisplatin treatment (Honma et al., 2008). In addition, gastric cancers with high RPN2 expression have exhibited dramatically higher recurrence rates and lower 5-year survival rates relative to those with low expression (Fujimoto et al., 2017). These observations suggest that RPN2 expression could serve as a predictive biomarker for chemotherapy resistance. In a recent study, RPN2 was reported to be modulated by circNFIX, and promoted GBM tumor growth in vivo and in vitro (Ding et al., 2019). However, the correlation of RPN2 expression and radiotherapy resistance in GBM remains unknown.

This study explored the function of RPN2 in radioresistant GBM, and found that its high expression contributes to the tolerance of GBM to radiotherapy. The dysregulation of RPN2 led to abnormal myeloid cell leukemia 1 (MCL1) expression through the promotion of STAT3 transcription activity. Our study, therefore, provides a new target to overcome radioresistance in GBM therapy.

\section{Methods}

\section{Bioinformatics analysis}

The abnormal expression of RPN2 and MCL1 was investigated through the UCSC Cancer Genomics Browser (https://xena.ucsc.edu/welcome-to-ucsc-xena/) and GEPIA online database (http://gepia.cancer-pku.cn/).

\section{Patient samples and cell culture}

GBM samples were taken from 34 patients admitted to the First Affiliated Hospital of Harbin Medical University. These GBM patients had all received radiation therapy, with 12 patients experiencing GBM recurrence. The corresponding brain samples were harvested and preserved at $-80^{\circ} \mathrm{C}$. Informed consent was obtained from all participants, and the study was approved by the Ethics Committee of the First Affiliated Hospital of Harbin Medical University.

The normal glioma cell lines (U87, T98, U251, U118MG and A172) and astrocyte cell line (HA) were provided by BeNa Culture Collection (Beijing, China). These cells were cultivated in DMEM (Sigma, St. Louis, $\mathrm{MO}$, USA) with $10 \% \mathrm{FBS}$ at $37^{\circ} \mathrm{C}$ under $5 \% \mathrm{CO}_{2}$.

\section{Radiotherapy}

Radiotherapy was conducted in the Radiotherapy Oncology department of the First Affiliated Hospital of Harbin Medical University, using a Varian 2100C linear accelerator (dose, $5 \mathrm{~Gy}$; dose rate, $5 \mathrm{~Gy} / \mathrm{min}$ ). The cells were seeded in a 12-well plate and preserved under adjustable conditions for 1 day, and subsequently treated with radiation, and cultivated again under identical conditions for 1 day more.

\section{Clonal radioresistant cell generation}

A172 and U87 cells were seeded in culture plates (100 $\mathrm{mm}$ ) and treated with a 1 Gy radiation dose until an accumulated dose of $5 \mathrm{~Gy}$ was reached. All dissociated cells were recovered using cloning cylinders (Sigma Aldrich) and seeded in a 96-well plate. Once proliferating, these cells were transferred to a 12-well plate, and subsequently a 6 -well plate. Colonel radioresistant cells were then obtained.

\section{Cell transfection}

U87 cells were inoculated in 12 -well plates $\left(1 \times 10^{5}\right.$ cells/ well). RPN2, MCL1, and STAT3 siRNA or control siRNA (GenePharma, Co., Ltd., Shanghai, China) were transfected into cells through Lipofectamine 2000 (Invitrogen, Shanghai, China) at 50-60\% confluency. Two days later, the resulting cells were harvested and preserved for future use. The siRNA sequences involved were: RPN2, 5' -GGCCACUGUUAAACUAGAACA-3'; MCL1, 5'-CGCCGAAUUCAUUAAUUUAUU-3'; STAT3, 5' CUUCAGACCCGUCAACAAA-3'. The negative (mock) siRNA sequence involved was $5^{\prime}$-ACGCCUCCCGAACG UTTUUCUUGUCGUC-3'. The pcDNA-V5-RPN2 was constructed by inserting the RPN2 cDNA into pcDNA 3.1 / V5-His TOPO vector (Thermofisher, Waltham, MA. USA) according to the manufacturer instructions.

\section{qPCR}

RNA was isolated using TRIzol (Thermo Fisher Scientific) and quantified using a NanoDrop 2000 (Thermo Fisher). RNA was treated with RNase R (Geneseed, Guangzhou, China) to achieve circRNA purification. The cDNA was prepared from $0.5 \mu \mathrm{g}$ RNA using a PrimeScript RT reagent kit (TaKaRa, Dalian, China) or TaqMan miRNA Reverse Transcription Kit (Applied Biosystems, FosterCity, CA, USA). qPCR was conducted using a PCR System (Bio-Rad). The following were primer sequences involved: RPN2 (Forward, 5' - AGGAAGTGGT GTTTGTTGCC-3'; Reverse, 5'-CAGTCGAGGGAGCT TCTTC-3'); MCL1 (5'-AAAGCCTGTCTGCCAAAT-3' and reverse primer $5^{\prime}$-CCTATAAACCCACCACTC-3'); and GAPDH (Forward, 5'-GAATGGGCAGCCGTTAGGAA-3'; Reverse, 5' -AAAAGCATCACCCGGAGGAG-3'). 


\section{Western blot (WB)}

Western blot analysis was conducted to isolate the target proteins using RIPA buffer (Beyotime) containing protease inhibitors. The protein concentrations were determined using a BCA Kit. Subsequently, the same amounts $(0.02 \mathrm{mg})$ of protein were treated with boiled water and isolated using the SDS-PAGE technique. The SDS-PAGE gels were subsequently transferred to $0.45 \mu \mathrm{m}$ PVDF membranes (Millipore, Billerica, MA, USA), which were exposed to TBST containing 5\% fatfree milk for 60 min and cultivated with RPN2, MCL1, BCL2, BAX, BIM (Abcam, Cambridge, MA, USA), NOXA, PUMA, cleaved caspase-3 (CASP3), STAT3, pSTAT3 and $\beta$-actin (ACTB) (Cell Signaling, Danvers, MA, USA) antibodies at $4{ }^{\circ} \mathrm{C}$ overnight. After washed with TBST for 3 times, and the membranes were cultivated with 2nd antibody (Abcam) diluted at 1:1000 for $120 \mathrm{~min}$ at room temperature.

Immunohistochemistry and immunofluorescence staining GBM tissues were fixed in 10\% formalin followed by paraffin embedding. Immunohistochemistry (IHC) was performed on $5 \mu \mathrm{m}$ tissue sections using the antibodies of RPN2, MCL1 (Abcam), p-STAT3 (Cell Signaling) according to manufacturer's instructions. The results of the IHC analysis were taken with a digital slide scanning system (Pannoramic Scan, 3DHISTECH Ltd.). The mean density (IOD/area) were quantified by Image J software (NIH, USA). The immunofluorescence staining of STAT3 and nuclear labeling with DAPI were performed as previously described (Bhattacharya et al., 2005).

\section{MTS and apoptosis assay}

Cells (5000) were inoculated in 96-well plates and cultivated at $37^{\circ} \mathrm{C}$ overnight in medium supplemented with $10 \%$ FBS. Following this, cell viability was determined using a cell proliferation kit (Promega, Madison, WI, USA). Each process was conducted in parallel at least three times, on three different occasions. The apoptosis analysis was conducted by using Hoechst33258 staining as described in the previous study (Chen et al., 2014). The results were expressed as average $\pm \mathrm{SD}$.

\section{Statistical analysis}

The experiments were conducted no less than three times, and the results presented as average \pm SD. Differences among groups were assessed using a Tukey test and $t$-test or with ANOVA, analyzed through Prism 7 (GraphPad Inc., La Jolla, CA, USA). Patients' overall survival $(\mathrm{OS})$ was obtained and evaluated using a $\mathrm{K}-\mathrm{M}$ plot and log-rank test, respectively.

\section{Results}

Abnormal expression of RPN2 is correlated to poor survival of GBM patients

To study the role of RPN2 in GBM radiotherapy, we first determined the expression of RPN2 in GBM by analyzing mRNA data in the Cancer Genome Atlas (TCGA) database. TCGA RNA-Seq data showed a marked upregulation of RPN2 in GBM tumor tissues compared to normal control tissues (Fig. 1a, b). Our analysis of GEPIA data indicated that high expression of RPN2 is associated with poorer survival in GBM patients, which strongly suggests that abnormal expression of $R P N 2$ plays an important role in GBM. Since radiotherapy is one of the primary treatment methods for GBM, we then analyzed RPN2 expression in different groups of GBM patients. We analyzed RPN2 expression in tissue samples obtained from 34 GBM patients who had received radiation treatment. Among these 34 patients, 12 patients had experienced GBM recurrence and were grouped as radioresistant patients. We then compared RPN2 expression levels in tumor samples from these 34 patients, with or without GBM recurrence. We found that the patients with tumor recurrence had higher RPN2 expression at the mRNA and protein level (Fig. 1c, d). Our data suggests that high RPN2 expression may contribute to radioresistance in GBM.

\section{RPN2 expression is higher in radioresistant GBM cells}

To study the role of RPN2 in radiotherapy resistant GBM, we generated radioresistant GBM cells by subjecting U87 and A172 cells lines to five successive rounds of radiation. We found that the $5 \mathrm{~Gy}$ radiation resulted in less cell death in resistant A172 and U87 cells, as analyzed by MTT assay (Fig. 2a). Apoptosis is the major cell death induced by radiation (Stupp et al., 2005). Therefore, we investigated the radiation triggered apoptosis in U87 and A172 cells using Hoechst 33258 staining, and observed that apoptosis was higher in parental cells than in the resistant cells (Fig. 2b). However, these cells did not display any differences in cell proliferation (Fig. 2c). We then analyzed the apoptotic signal in parental and resistant cells by analyzing cleaved CASP3 expression, and found that cleaved CASP3 was induced in A172 and U87 parental cells, while compromised in resistant cells (Fig. 2d). We then investigated the RPN2 expression in these cells and observed that resistant cells exhibited higher expression at the protein and mRNA level (Fig. 2d, e). Furthermore, the expression of RPN2 in GBM cells was also higher than that in the normal astrocyte cell line (HA) (Fig. 2f, g). Therefore, our results suggest that high expression of RPN2 could be correlated with radioresistance in GBM. 


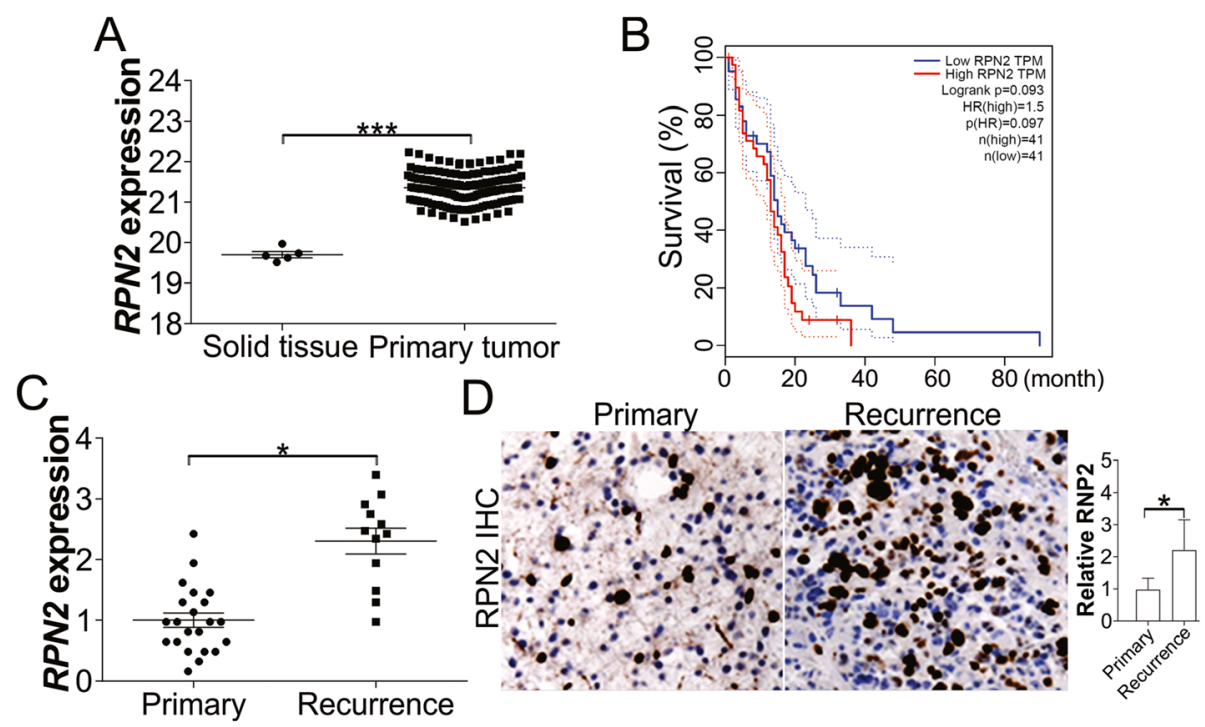

Fig. 1 High RPN2 expression in GBM is correlated to poor survival and recurrence. a The mRNA expression of RPN2 in solid tissue and primary GBM tumor based on database from cancer browser (https://xenabrowser.net/heatmap/). b The correlation of RPN2 expression with GBM patient survival based on the database of GEPIA (http://gepia2.cancer-pku.cn/\#index). c The mRNA expression of RPN2 in 34 GBM patients with radiation therapy history. 12 of them had GBM recurrence. $\mathbf{d}$ The IHC staining of RPN2 in primary and recurrence GBM tumors. Left, the representative pictures; right, the histological analysis of RPN2 expression by Image J. ${ }^{*}, p<0.05 ;{ }^{* * *}, p<0.001$

Expression of RPN2 contributes to radioresistance in GBM To confirm whether high expression of RPN2 is necessary for radioresistance in GBM, siRNA-mediated knockdown of RPN2 was carried out in U87 and A172 resistant cells. It was observed that RPN2 deprivation resensitized the $\mathrm{U} 87$ and $\mathrm{A} 172$ resistant cells to $5 \mathrm{~Gy}$ radiation induced cell death (Fig. 3a). In terms of apoptosis, absence of RPN2 enhanced radiation induced apoptosis (Fig. 3b, c). Conversely, enhanced expression of RPN2 in U87 parental cells had the opposite effect on radiation induced cell death, including the suppression of cell loss (Fig. 3d), prevention of apoptosis (Fig. 3e), and reduction of CASP3 cleavage (Fig. 3f). Therefore, our data indicates that RPN2 expression contributes to radioresistance in GBM cells.

\section{RPN2 regulated the MCL1 expression in radio-resistant GBM cells}

We then investigated the mechanisms underlying the role of RPN2 in GBM radioresistance. The expression of Bcl-2 family proteins is critical for radiation induced apoptosis, and the dysregulation of these proteins usually leads to radioresistance. The expression of several Bcl-2 family proteins was assessed in U87 parental, resistant cells, and U87 parental cells with pcDNA-RPN2 transfection. The western blot revealed that only MCL1 was upregulated in U87 resistant cells, and in U87 parental cells with RPN2 overexpression (Fig. 4a). However, other Bcl-2 family proteins, including BCL2, BCL2L1, BAX, BIM, NOXA, and PUMA, did not show any changes (Fig. 4a). In contrast, depletion of RPN2 by siRNA suppressed MCL1 expression in U87 resistant cells (Fig. 4b). MCL1 mRNA levels were also consistently upregulated in U87 parental cells with RPN2 overexpression, and downregulated in RPN2 knockdown U87 resistant cells (Fig. 4c). To further confirm the relationship between RPN2 and MCL1, we analyzed MCL1 expression in the Cancer Genome Atlas (TCGA) database, and found that MCL1 expression was also higher in GBM primary tumors (Fig. 4d), and positively correlated with the expression of RPN2 (Fig. 4e). To further study the role of MCL1 dysregulation in radioresistant GBM, siRNA-mediated knockdown of MCL1 was carried out by transfecting U87 and A172 resistant cells. Silencing of MCL1 had a similar effect to that of RPN2 knockdown, which enhanced the expression of CASP3, and nuclear fragmentation caused by radiation (Fig. 4f, g). However, absence of MCL1 did not affect the expression of RPN2 (Fig. 4f). Similarly, pretreatment with an MCL1 inhibitor, ABT-737, resulted in a similar effect, sensitizing the radioresistant U87 and A172 cells to radiation triggered apoptosis (Fig. 4h). The bliss synergy score of ABT737 with irradiation in these two cells were 2.09 and 1.65, respectively. The histological analysis of MCL1 expression by IHC revealed that MCL1 expression in the recurrent GBM was also higher than the primary tumors (Fig. 4i). Taken together, these findings show that RPN2 promotes radioresistance in GBM by upregulating MCL1 at the mRNA level. 

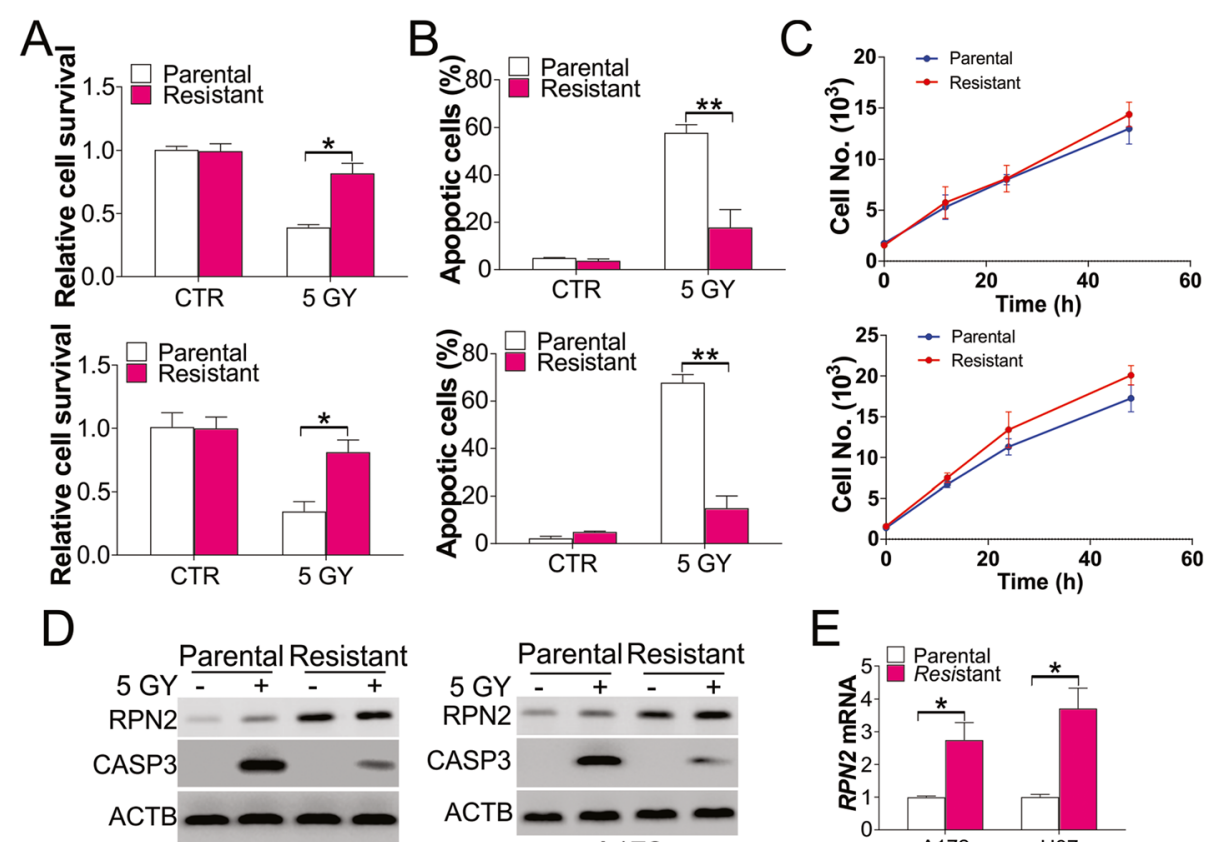

$\mathrm{E}$
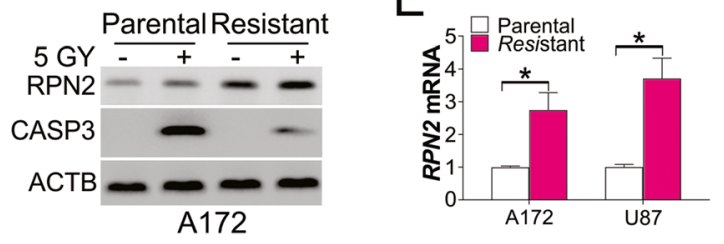

$\mathrm{F}$

U87

G

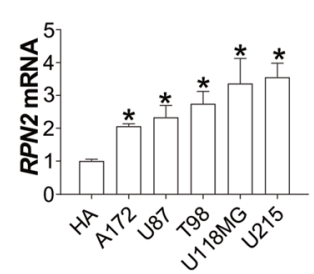

Fig. 2 RPN2 was upregulated in radioresistant GBM cells. a The survival of parental and radioresistant U87 (upper) and A172 (lower) cells treated with $5 \mathrm{GY}$ radiation. b The apoptosis of parental and radioresistant U87 (upper) and A172 (lower) cells treated with 5 GY radiation. c The growth of parental and radioresistant U87 (upper) and A172 (lower) cells. $\mathbf{d}$ The expression of RPN2 and CASP3 in parental and radioresistant U87 (left) and A172 (right) cells treated with 5 GY radiation. e The mRNA level of RPN2 in parental and radioresistant U87 and A172 cells. f, $\mathbf{g}$ The protein (f) and mRNA (g) level of RPN2 in the indicated cells. Each experiment was performed for 3 times. ${ }^{*}, p<0.05 ;{ }^{* *}, p<0.01$

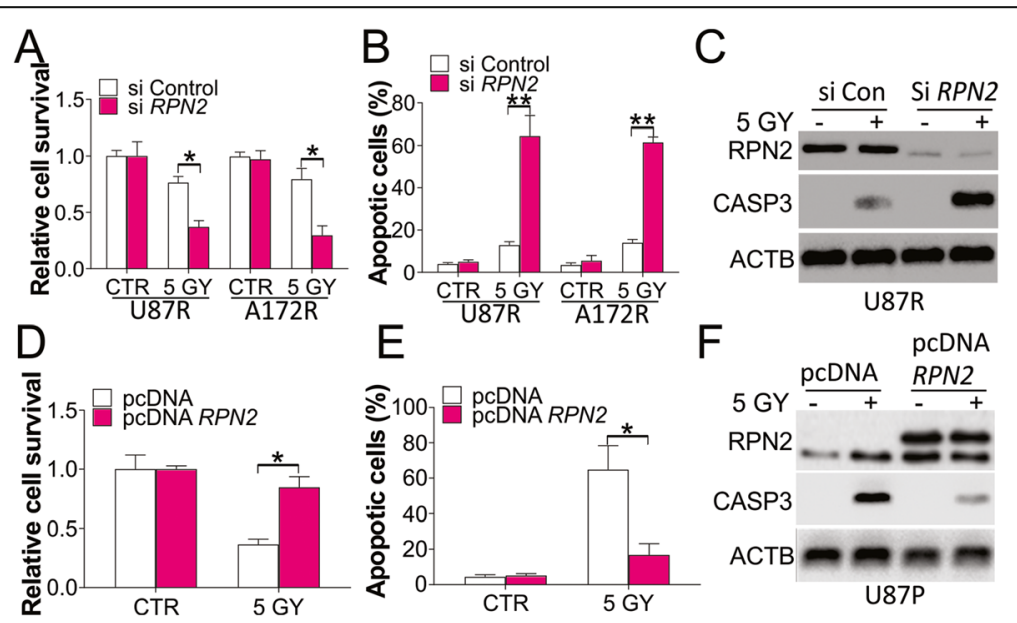

Fig. 3 Abnormal expression of RPN2 leads to radioresistance in GBM cells. a U87 and A172 resistant cells transfected with control or RPN2 siRNA were treated with $5 \mathrm{GY}$ radiation. The survival was analyzed by MTS assay. $\mathbf{b}$ The apoptosis of U87 and A172 resistant cells treated as in (a). c The expression of RPN2 and CASP3 in U87 resistant cells treated as in (a). $\mathbf{d}$ U87 parental cells transfected with control or RPN2 plasmids were treated with 5 GY radiation. The survival was analyzed by MTS assay. e The apoptosis of U87 parental cells treated as in (d). $\mathbf{f}$ The expression of RPN2 and CASP3 in U87 parental cells treated as in (d). Each experiment was performed for 3 times. ${ }^{*}, p<0.05 ;{ }^{* *}, p<0.01$ 

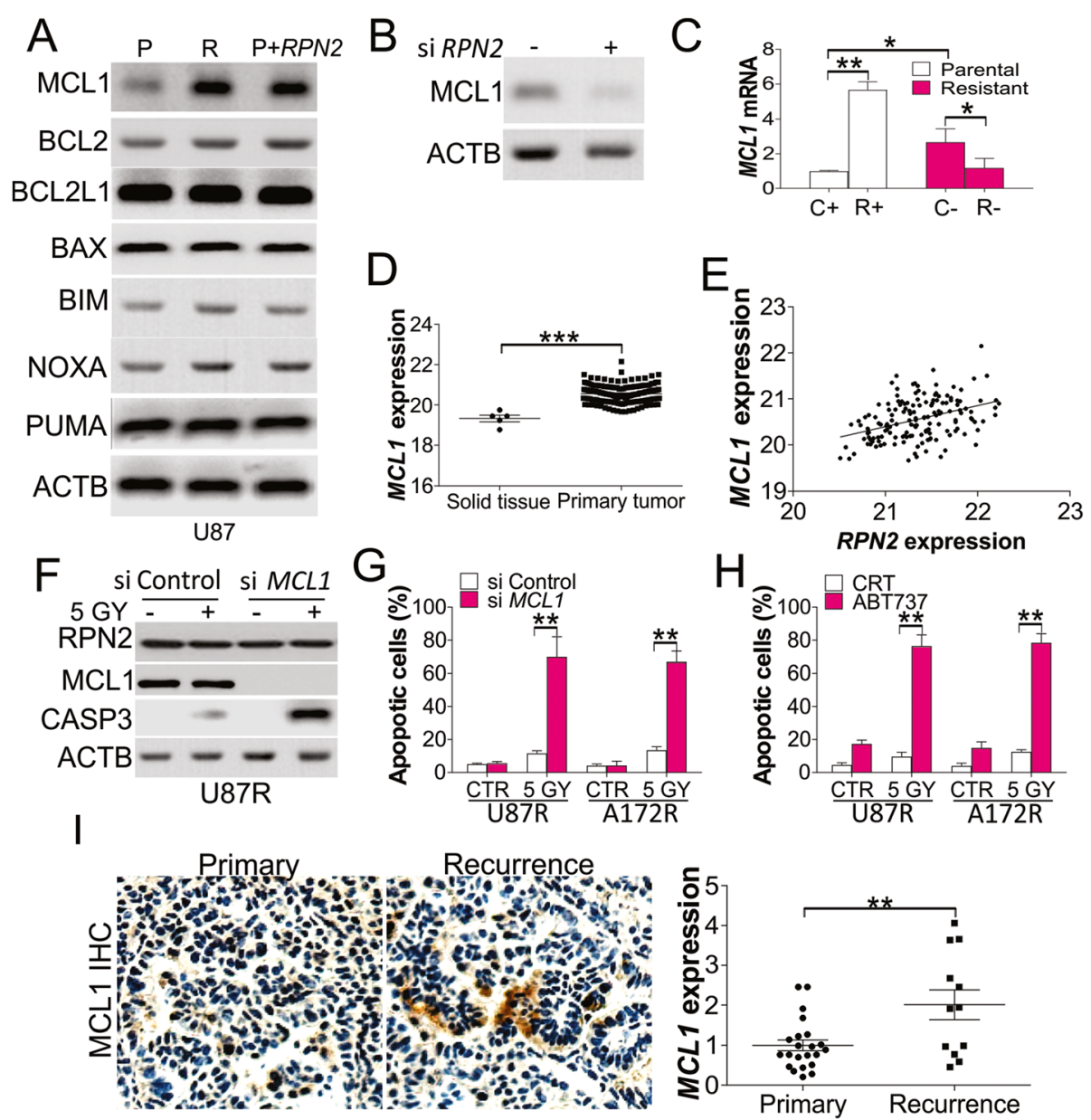

Fig. 4 RPN2 promotes MCL1 expression to mediate the radioresistance in GBM cells. a The expression of Bcl-2 family proteins in U87 parental, resistant cells, or U87 parental cells with RPN2 overexpression. b The expression of MCL1 in U87 resistant cells transfected with RPN2 siRNA. c The mRNA level of MCL1. C+: pcDNA transfection; R+: pcDNA RPN2 transfection; C-: si control transfection; R-: si RPN2 trasnfection. $\mathbf{d}$ The expression level of MCL1 in GBM tumors based on the database of cancer browser (https://xenabrowser.net/heatmap/). e The pearson correlation of RPN2 and MCL1 from database of cancer browser. $R^{2}=0.1502, p<0.001$. $\mathbf{f} \cup 87$ resistant cells transfected with MCL1 siRNA were treated with 5 GY radiation. The expression of RPN2, MCL1 and CASP3 was analyzed by western blot. $\mathbf{g}$ The apoptosis of U87 and A172 resistant cells treated as in (f). $\mathbf{h}$ The apoptosis of U87 and A172 resistant cells treated with ABT-737 $(5 \mu \mathrm{M})$ in combination with 5 GY radiation. $\mathbf{i}$ The IHC staining of MCL1 in primary and recurrence GBM tumors. Left, the representative pictures; right, the histological analysis of MCL1 expression by Image J. Each experiment was performed for 3 times. ${ }^{*}, p<0.05 ;{ }^{* *}, p<0.01 ;{ }^{* * *}, p<0.001$

RPN2 modulated MCL1 expression by stimulating STAT3 In the next stage of this study, we assessed how RPN2 modulates the expression of MCL1 in radioresistant GBM. Previous studies have shown that MCL1 expression is regulated via STAT3 phosphorylation (Bhattacharya et al., 2005), which was recently reported to be activated by RPN2 (Ni et al., 2020). We therefore studied STAT3 activation levels upon RPN2 overexpression. As expected, RPN2 overexpression improved STAT3 phosphorylation levels without influencing its expression (Fig. 5a). And the STAT3 phosphorylation also increased the U87 resistant cells when compared with the parental cells (Fig. 5a). Furthermore, overexpression of RPN2 also enhanced the nuclear translocation of STAT3 (Fig. 5b), suggesting that RPN2 expression activates STAT3. To determine the role of STAT3 in RPN2-triggered MCL1 upregulation, we depleted STAT3 in U87 and A172 resistant cells, and found that silencing of STAT3 inhibited MCL1 expression at the protein and mRNA level (Fig. 5c, d). Silence of STAT3 did not affect the expression of RPN2 (Fig. 5c). Depletion of STAT3 sensitized U87 resistant cells to radiation induced apoptosis (Fig. 5c, e). Moreover, the phosphorylation of STAT3 was higher in the recurrent GBM tissues than the primary tumors (Fig. 5f). These findings confirm that the STAT3 pathway is associated with RPN2-modulated MCL1 expression in radioresistant GBM cells.

\section{Discussion}

The RPN2 gene has been localized to chromosome 20ql2-13.1, a region that is often compromised in 

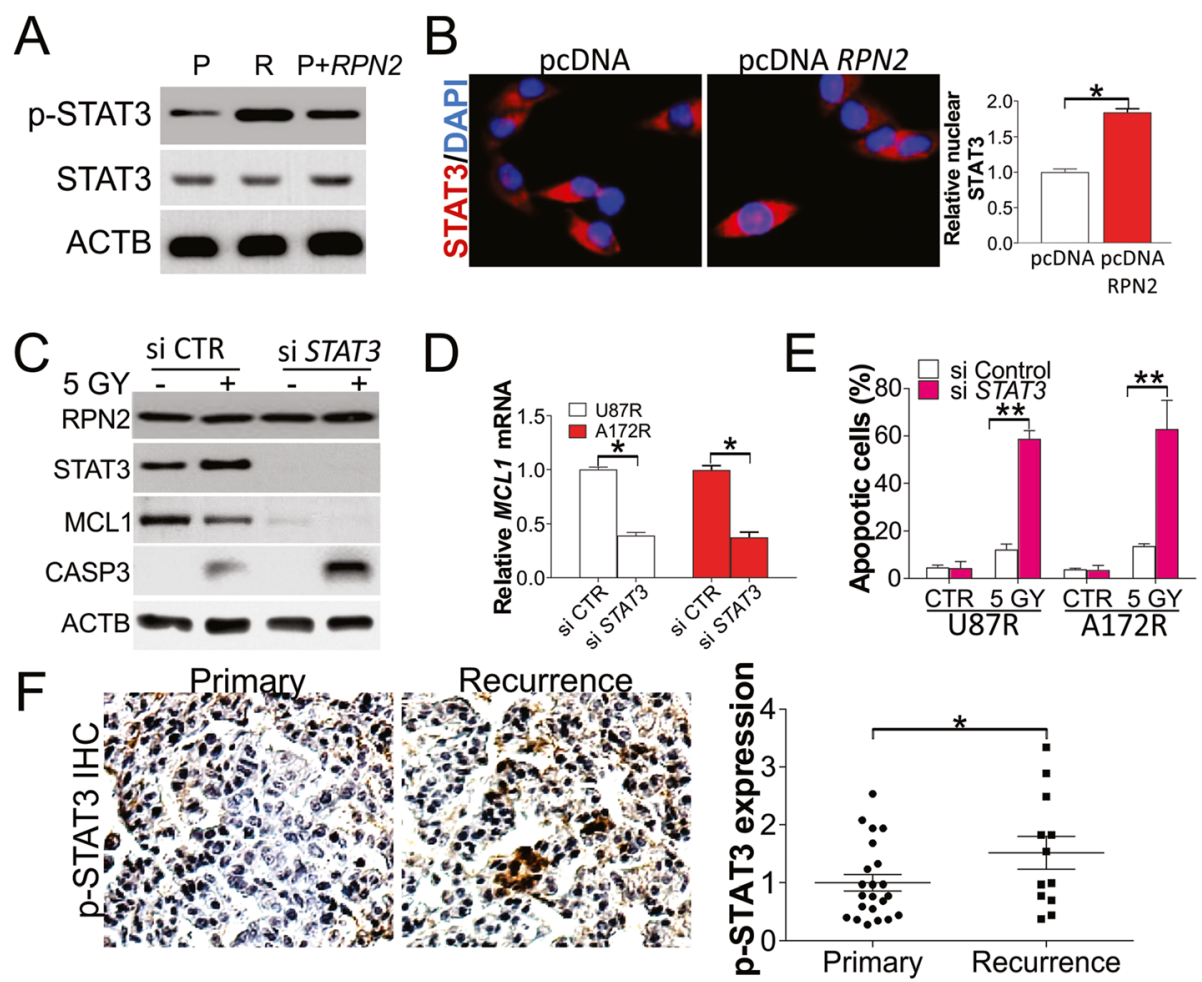

Fig. 5 RPN2 mediates MCL1 expression by activate STAT3. a The expression of p-STAT3 and STAT3 in U87 parental, resistant cells, or U87 parental cells with RPN2 overexpression. $\mathbf{b}$ The immunofluorescence staining of STAT3 in U87 cells transfected with RPN2 plasmid. Left, the representative pictures; right, the nuclear translocation of STAT3 was analyzed by Image J. c U87 resistant cells transfected with STAT3 siRNA were treated with 5 GY radiation. The expression of RNP2, MCL1, STAT3, and CASP3 was analyzed by western blot. $\mathbf{d}$ The mRNA level of MCL1 in U87 and A172 resistant cells transfected with STAT3 siRNA. e The apoptosis of U87 and A172 resistant cells treated as in (c). $\mathbf{f}$ The IHC staining of p-STAT3 in primary and recurrence GBM tumors. Left, the representative pictures; right, the histological analysis of p-STAT3 expression by Image J. Each experiment was performed for 3 times. ${ }^{*}, p<0.05 ;{ }^{*}, p<0.01$

patients who develop malignant myeloid tumors (Davis et al., 1984; Loffler et al., 1991). Besides its correlation with myeloid disturbance, RPN2 has also been identified as an indicator of prognosis in human pancreatic and breast cancer (Kaushal et al., 2012; Zhu et al., 2012). It has also been associated with the resistance of tumor cells to chemotherapeutic drugs in the breast (Honma et al., 2008) and ovarian (De Souza et al., 2011) cancers, and esophageal squamous cell carcinomas (Kurashige et al., 2012). Moreover, shRNAs-triggered RPN2 silencing has been shown to suppress lung tumor formation and increase cancer cell sensitivity to cisplatin (Fujita et al., 2015). In another study, RPN2 mediated chemosensitivity was mediated by $\mathrm{P}$-gp protein expression, which was downregulated after cisplatin treatment in gastric cancer cells without RPN2 (Yuan et al., 2015). However, the activity of RPN2 in response to different doses of ionizing radiation has not been investigated. This study has revealed a strong correlation between an increase in RPN2 expression and radioresistance in GBM in vitro and in vivo. We found that abnormal RPN2 expression promotes MCL1 expression by stimulating STAT3, thereby promoting radioresistance (Fig. 6).

The activation of STAT3 plays a vital role in mediating anti-apoptosis genes to suppress cell apoptosis, including MCL1 (Bhattacharya et al., 2005). It was reported that activation of STAT3 suppressed cell apoptosis, and promoted cell migration and invasion, cell cycle arrest by modulating several genes' expression in liver, ovarian and colon cancer (Bhattacharya et al., 2005; Chung et al., 1997; Huang et al., 2019). In our study, we found that RPN2 overexpression enhanced the phosphorylation levels STAT3 and promoted its nuclear translocation (Fig. 5), suggesting the activation of STAT3 by enhanced RPN2 expression. Consistent with our study, the activation of STAT3 by RPN2 was found in other types of cancers, including colon carcinoma, hepatocellular carcinoma (Huang et al., 2019; Bi \& Jiang, 2018). RPN2 was reported to regulate colon cancer cell proliferation through mediating the glycosylation of EGFR, which affectes the EGFR/ERK signaling pathways ( $\mathrm{Li}$ et al., 2017). Since EGFR/ERK can directly phosphorylate and activate STAT3 (Chung et al., 1997), we hypothesized 


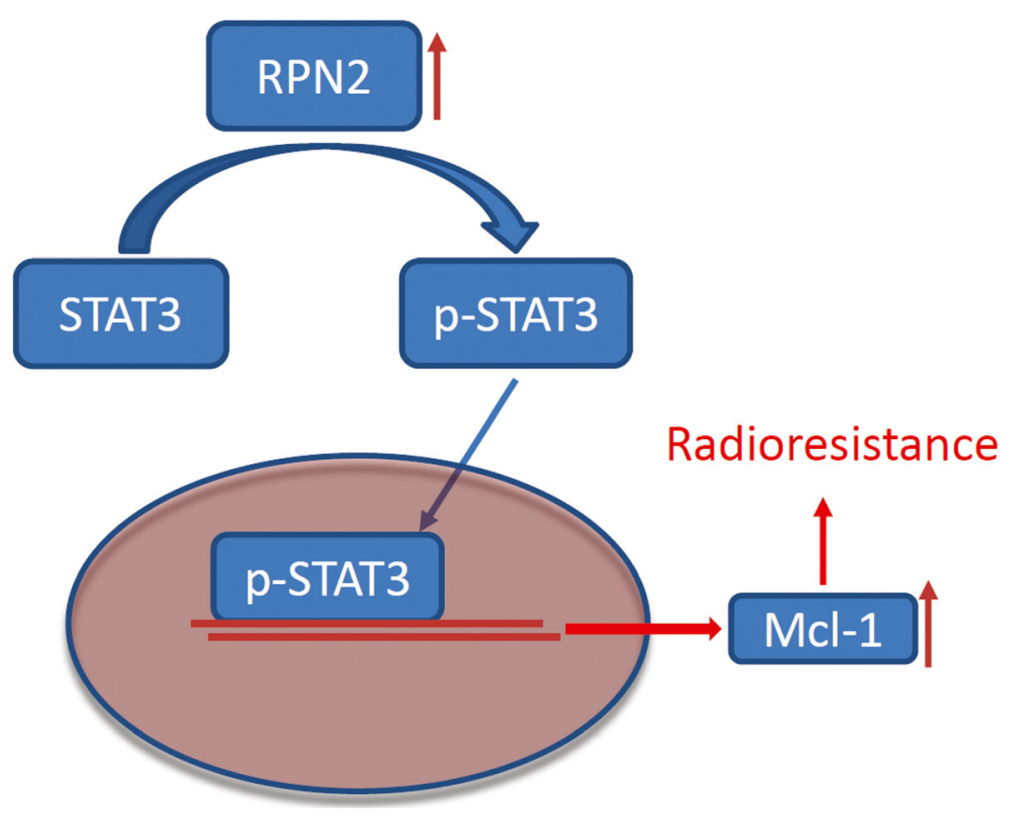

Fig. 6 The graphic summary of RPN2/STAT3/MCL1 pathway

that RPN2 might modulate the activity of STAT3 by EGFR glycosylation. However, the exact mechanism still needs further investigation.

Apoptosis triggered by DNA damage is the primary mechanism of action in radiotherapy (Norbury \& Zhivotovsky, 2004). Abnormal Bcl-2 family protein expression is frequently detected in multiple cancers (Davids \& Letai, 2012), and contributes to radio-resistance both in vitro and in vivo (Chen et al., 2018). Radioresistance may also result from perturbations in homologous recombination repair and DNA double-strand break repair, by error-prone and alternative end-joining. Furthermore, genetic and pharmacologic targeting of MCL1 or BCL2L1 has been shown to effectively improve the sensitivity of cancer cells to radiotherapy (Chen et al., 2018). The complicated molecular interplay of pro-survival and pro-death Bcl-2 members determinants cell survival and apoptosis rate. Alterations in this balance resulting from responses to survival stimuli is only just starting to be understood. Lopez et al displayed that apoptosis triggered by DNA damage occurs when MCL1 and BCL2L1 are suppressed (Lopez et al., 2010). Fujita et al confirmed RPN2 facilitated cell proliferation and repression of apoptosis by modulating BAX/BCL2 in non-small cell lung cancer (Fujita et al., 2015). However, we did not find that the expression of BCL2 changed with RPN2 expression in this study. Instead we found that MCL1 expression was up-regulated in radioresistant and RPN2 overexpressed GBM cells, and appears to contribute to the tolerance of radiation. Repressing MCL1 may play clinical role in treating diverse radioresistant tumors, although the exact pharmacological mechanism of MCL1 suppression has not yet been established. Some pan-Bcl-2 inhibitors were prepared, such as ABT-737, and can suppress tumor formation and survival (Davids \& Letai, 2012; Thomas et al., 2013). Here we show that administration of ABT-737 can overcome the radioresistance in RPN2 overexpressed GBM cells, suggesting that inhibition of MCL1 could be a therapy strategy for GBM patients with abnormal tumor RPN2 expression.

\section{Conclusions}

In conclusion, this study has shown an obvious distinction in overall survival between RPN2 high expression and low expression GBM patients, as well as a correlation between RPN2 expression and the effectiveness of radiation. This study is the first to indicate that RPN2 expression may serve as a predictive biomarker for radiation therapy in GBM patients.

\section{Abbreviations}

GBM: Glioblastoma; RPN2: Ribophorin II; MCL1: Myeloid cell leukemia 1; WB: Western blot; OS: Overall survival; TCGA: The Cancer Genome Atlas

\section{Acknowledgements}

Not applicable.

\section{Authors' contributions}

LCY, RHN and SB designed experiments; SSJ and LWS carried out experiments; ZWH, JB and ZHM analyzed experimental results. LCY and RHN wrote the manuscript, SB revise the manuscript. All authors approved the final manuscript.

\section{Funding}

This work was supported by Key R \& D Plan of Hainan Province and Finance Science and Technology Project of Hainan Province (No. ZDYF2019181). 


\section{Availability of data and materials}

Not applicable.

\section{Ethics approval and consent to participate}

Informed consent was obtained from all participants and the study was approved by the Ethics Committee of the First Affiliated Hospital of Harbin Medical University.

\section{Consent for publication}

Not applicable.

\section{Competing interests}

The authors declare that they have no competing interests.

\section{Author details}

${ }^{1}$ Neurosurgery, Hainan Cancer Hospital, Haikou, China. ${ }^{2}$ Radiotherapy Department, Hainan Cancer Hospital, Haikou, China. ${ }^{3}$ Head and Neck Surgery, Hainan Cancer Hospital, Haikou, China. ${ }^{4}$ Hematology Department, Hainan Cancer Hospital, Haikou, China. ${ }^{5}$ Clinical Pharmacy Department, Hainan Cancer Hospital, Haikou, China. ${ }^{6}$ Neurosurgery, The First Affiliated Hospital of Harbin Medical University, No.199 Dazhi Street, Nangang District, Harbin 150001, Heilongjiang, China.

Received: 3 March 2020 Accepted: 23 April 2020

Published online: 13 May 2020

\section{References}

Bhattacharya S, Ray RM, Johnson LR. STAT3-mediated transcription of BCl-2, Mcl-1 and c-IAP2 prevents apoptosis in polyamine-depleted cells. Biochem J. 2005; 392:335-44.

Bi C, Jiang B. Downregulation of RPN2 induces apoptosis and inhibits migration and invasion in colon carcinoma. Oncol Rep. 2018;40:283-93.

Chen D, Ming L, Zou F, Peng Y, Van Houten B, Yu J, Zhang L. TAp73 promotes cell survival upon genotoxic stress by inhibiting p53 activity. Oncotarget. 2014:5:8107-22

Chen G, Magis AT, Xu K, Park D, Yu DS, Owonikoko TK, Sica GL, Satola SW, Ramalingam SS, Curran WJ, Doetsch PW, Deng X. Targeting Mcl-1 enhances DNA replication stress sensitivity to cancer therapy. J Clin Invest. 2018;128: 500-16.

Chung J, Uchida E, Grammer TC, Blenis J. STAT3 serine phosphorylation by ERKdependent and -independent pathways negatively modulates its tyrosine phosphorylation. Mol Cell Biol. 1997;17:6508-16.

Davids MS, Letai A. Targeting the B-cell lymphoma/leukemia 2 family in cancer. J Clin Oncol. 2012:30:3127-35.

Davis MP, Dewald GW, Pierre RV, Hoagland HC. Hematologic manifestations associated with deletions of the long arm of chromosome 20. Cancer Genet Cytogenet. 1984;12:63-71.

De Souza R, Zahedi P, Badame RM, Allen C, Piquette-Miller M. Chemotherapy dosing schedule influences drug resistance development in ovarian cancer. Mol Cancer Ther. 2011;10:1289-99.

Ding C, Wu Z, You H, Ge H, Zheng S, Lin Y, Wu X, Lin Z, Kang D. CircNFIX promotes progression of glioma through regulating miR-378e/RPN2 axis. J Exp Clin Cancer Res. 2019;38:506.

Fujimoto D, Goi T, Hirono Y. Expression of ribophorine II is a promising prognostic factor in human gastric adenocarcinoma. Int J Oncol. 2017;50: 448-56.

Fujita Y, Yagishita S, Takeshita F, Yamamoto Y, Kuwano K, Ochiya T. Prognostic and therapeutic impact of RPN2-mediated tumor malignancy in non-smallcell lung cancer. Oncotarget. 2015;6:3335-45.

Ghotme KA, Barreto GE, Echeverria V, Gonzalez J, Bustos RH, Sanchez M, Leszek J, Yarla NS, Gomez RM, Tarasov W, Ashraf GM, Aliev G. Gliomas: new perspectives in diagnosis, treatment and prognosis. Curr Top Med Chem. 2017:17:1438-47.

Hess KR, Broglio KR, Bondy ML. Adult glioma incidence trends in the United States, 1977-2000. Cancer. 2004;101:2293-9.

Honma K, Iwao-Koizumi K, Takeshita F, Yamamoto Y, Yoshida T, Nishio K, Nagahara S, Kato K, Ochiya T. RPN2 gene confers docetaxel resistance in breast cancer. Nat Med. 2008;14:939-48.

Huang L, Jian Z, Gao Y, Zhou P, Zhang G, Jiang B, Lv Y. RPN2 promotes metastasis of hepatocellular carcinoma cell and inhibits autophagy via STAT3 and NF-kappaB pathways. Aging (Albany NY). 2019;11:6674-90.
Kaushal M, Mishra AK, Sharma J, Zomawia E, Kataki A, Kapur S, Saxena S. Genomic alterations in breast cancer patients in betel quid and non betel quid chewers. PLoS One. 2012;7:e43789.

Kurashige J, Watanabe M, Iwatsuki M, Kinoshita K, Saito S, Nagai Y, Ishimoto T, Baba Y, Mimori K, Baba H. RPN2 expression predicts response to docetaxel in oesophageal squamous cell carcinoma. Br J Cancer. 2012;107:1233-8.

Li H, Al-Japairai K, Tao Y, Xiang Z. RPN2 promotes colorectal cancer cell proliferation through modulating the glycosylation status of EGFR. Oncotarget. 2017;8:72633-51.

Loffler C, Rao W, Hansmann I. Mapping of the ribophorin II (RPN II) gene to human chromosome 20q12-q13.1 by in-situ hybridization. Hum Genet. 1991; 87:221-2.

Lopez H, Zhang L, George NM, Liu X, Pang X, Evans JJ, Targy NM, Luo X. Perturbation of the $\mathrm{BCl}-2$ network and an induced $\mathrm{Noxa} / \mathrm{BCl}-\mathrm{xL}$ interaction trigger mitochondrial dysfunction after DNA damage. J Biol Chem. 2010;285: 15016-26.

Mirimanoff RO, Gorlia T, Mason W, Van den Bent MJ, Kortmann RD, Fisher B, Reni M, Brandes AA, Curschmann J, Villa S, Cairncross G, Allgeier A, Lacombe D, Stupp R. Radiotherapy and temozolomide for newly diagnosed glioblastoma: recursive partitioning analysis of the EORTC 26981/22981-NCIC CE3 phase III randomized trial. J Clin Oncol. 2006;24:2563-9.

Mrugala MM, Chamberlain MC. Mechanisms of disease: temozolomide and glioblastoma--look to the future. Nat Clin Pract Oncol. 2008;5:476-86.

Ni L, Yu J, Gui X, Lu Z, Wang X, Guo H, Zhou Y. Overexpression of RPN2 promotes osteogenic differentiation of hBMSCs through the JAK/STAT3 pathway. FEBS Open Bio. 2020:10:158-67.

Norbury CJ, Zhivotovsky B. DNA damage-induced apoptosis. Oncogene. 2004;23: 2797-808.

Perazzoli G, Prados J, Ortiz R, Caba O, Cabeza L, Berdasco M, Gonzalez B, Melguizo C. Temozolomide resistance in Glioblastoma cell lines: implication of MGMT, MMR, P-glycoprotein and CD133 expression. PLoS One. 2015;10: e0140131.

Preusser M, de Ribaupierre S, Wohrer A, Erridge SC, Hegi M, Weller M, Stupp R. Current concepts and management of glioblastoma. Ann Neurol. 2011;70:921.

Stupp R, Mason WP, van den Bent MJ, Weller M, Fisher B, Taphoorn MJ, Belanger K, Brandes AA, Marosi C, Bogdahn U, Curschmann J, Janzer RC, Ludwin SK, Gorlia T, Allgeier A, Lacombe D, Cairncross JG, Eisenhauer E, Mirimanoff RO. European organisation for $\mathrm{R}$, treatment of Cancer brain $\mathrm{T}$, radiotherapy $\mathrm{G}$ and National Cancer Institute of Canada clinical trials G. radiotherapy plus concomitant and adjuvant temozolomide for glioblastoma. N Engl J Med. 2005:352:987-96.

Thomas S, Quinn BA, Das SK, Dash R, Emdad L, Dasgupta S, Wang XY, Dent P, Reed JC, Pellecchia M, Sarkar D, Fisher PB. Targeting the BCl-2 family for cancer therapy. Expert Opin Ther Targets. 2013;17:61-75.

Yuan TM, Liang RY, Chueh PJ, Chuang SM. Role of ribophorin II in the response to anticancer drugs in gastric cancer cell lines. Oncol Lett. 2015;9:1861-8.

Zhu J, He J, Liu Y, Simeone DM, Lubman DM. Identification of glycoprotein markers for pancreatic cancer CD24+CD44+ stem-like cells using nano-LCMS/MS and tissue microarray. J Proteome Res. 2012;11:2272-81.

\section{Publisher's Note}

Springer Nature remains neutral with regard to jurisdictional claims in published maps and institutional affiliations.

Ready to submit your research? Choose BMC and benefit from:

- fast, convenient online submission

- thorough peer review by experienced researchers in your field

- rapid publication on acceptance

- support for research data, including large and complex data types

- gold Open Access which fosters wider collaboration and increased citations

- maximum visibility for your research: over $100 \mathrm{M}$ website views per year

At $\mathrm{BMC}$, research is always in progress.

Learn more biomedcentral.com/submission 